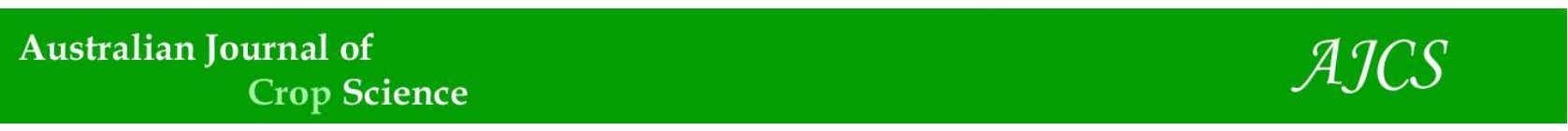

AJCS 15(08):1164-1171 (2021)

ISSN:1835-2707

doi: 10.21475/ajcs.21.15.08.p3228

\title{
Linear relationships between grain yield and tassel traits in maize
}

\section{Daniela Lixinski Silveira ${ }^{1^{*}}$, Alberto Cargnelutti Filho ${ }^{2}$, Cleiton Antônio Wartha ${ }^{3}$, Fernanda Carini ${ }^{1}$, Cirineu Tolfo Bandeira ${ }^{1}$, Rafael Vieira Pezzini ${ }^{1}$, Felipe Manfio Somavilla ${ }^{4}$}

\author{
${ }^{1}$ Graduate Program in Agronomy, Federal University of Santa Maria, UFSM, Santa Maria, RS, Brazil \\ ${ }^{2}$ Department of Plant Sciences, Center of Rural Sciences (CCR), Federal University of Santa Maria, UFSM, 97105-900, Santa \\ Maria, RS, Brazil \\ ${ }^{3}$ Graduate Program in Applied Plant Sciences, University of Minnesota, Saint Paul, United States of America \\ ${ }^{4}$ Undergraduate Agronomy, Federal University of Santa Maria, Santa Maria, RS, Brazil
}

\section{*Corresponding author: danilisil@hotmail.com}

\begin{abstract}
This study aimed to verify the linear relationships between maize grain yield and tassel traits, identify traits for selection through direct and indirect effects over three crop years. Three experiments were performed with 20 maize genotypes in a randomized complete block design with three replicates. Individual variance analysis showed significant genotype effects for all traits in the three crop years. However, a joint analysis demonstrated a significant genotype effect at $5 \%$ error probability for all traits, but branching space length. Furthermore, only the number of secondary branches and tassel branch number did not show significant genotype $\times$ crop year interaction. These results revealed a genotype differentiated behavior over the evaluated years, emphasizing the need to evaluate genotypes in more than one crop year. Based on phenotypic and genotypic correlation matrices and path analyses, the number of secondary tassel branches showed a positive linear relationship with grain yield; therefore, it can be used for the indirect selection of maize plants in breeding programs.
\end{abstract}

Keywords: Zea mays L., multicollinearity, path analysis, indirect selection.

Abbreviations: CV_Coefficient of variation; Fc_F calculated for genotype; SA_Selective accuracy; PL_Peduncle length (considering the distance between the collar of the flag leaf and the first branch), in cm; BSL_Branching space length, in cm; CSL_Central spike length, in cm; TL_Tassel length, in cm; NPB_Number of primary branches; NSB_Number of secondary branches; TBN_Tassel branch number; PDM_Peduncle dry matter (considering the region between the flag leaf collar and the first branch), in g; BSDM_Branching space dry matter, in g; CSDM_Central spike dry matter, in g; TDM_Tassel dry matter, in g; GY_Grain yield, in the plot and expressed as $\mathrm{Mg}$ ha $^{-1}$ at $13 \%$ moisture; rp_Correlation

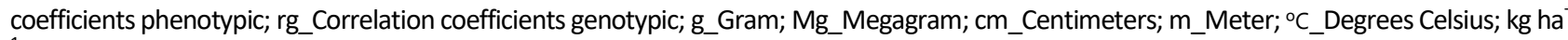
${ }^{1}$ _Kilogram per hectare; N_Nitrogen; $\mathrm{K}_{2} \mathrm{O} \_$Potassium monoxide; $\mathrm{P}_{2} \mathrm{O}_{5}$ _Phosphorus pentoxide; Oct_October; Nov_November; FEPAGRO_State Foundation for Agricultural Research of the state of Rio Grande do Sul; CN_Condition number; VIF_Variance inflation factor

\section{Introduction}

Maize (Zea mays L.) is a cereal grain used in several sectors of the production chain, from the production of staple foods such as flour, hominy, and oils to its use in the formulation of glucose syrup, maltodextrins, and dyes. Due to its socioeconomic importance, breeding studies have been carried out on grain yield as one of the main components for cultivar selection (Porto et al., 2011).

Maize breeding programs have sought to develop inbred lines and high-yielding hybrids with smaller tassel sizes (length) and fewer branches, but maintaining enough pollen production to favor fertilization (Duvick 2005; Fischer and Edmeades 2010). According to Edwards (2011) and Brewbaker (2015), plants with larger tassels inhibit solar radiation passage through the plant canopy, reducing photosynthetic activity and acting as a drain of photoassimilates, which may reduce grain yield.

In a study of a long-term commercial plant-breeding program, Duvick et al. (2004) reported negative linear regression coefficients for tassel-branch number, tassel weight, and tassel size score on released high-yielding U.S. maize hybrids with respective coefficients of determination $\left(R^{2}\right)$ of $0.66,0.70$, and
0.90. Moreover, a reduction in tassel size was sorted as a promoter of grain production efficiency intrinsic to maize plants exposed to intense selection for higher and more stable yields. However, Brewbaker (2015) concluded that there is little evidence that reductions in tassel size would result in higher grain yield, besides that tassels cannot become much smaller. Meanwhile, modern maize breeders are aware of increased tassel sizes in male inbred lines for greater pollen production and dispersal to assure the economic viability of hybrid seed production (Gage et al., 2018). Thus, studies of linear associations based on phenotypic and genotypic correlation coefficients can be used to measure the degree of association among traits. Linear correlations are valuable for selecting low-heritability and difficult-to-measure traits of agronomic interest.

A linear association among traits is used to quantify the magnitude and direction of influences but it does not allow assessing direct and indirect effects. Meantime, the correlation coefficients are unfolded into direct and indirect effects in path analysis, allowing to measure the influence of one variable on another, independently of the others (Cruz et al., 2014). Studies 
on correlations and path analysis involving maize tassel traits were developed (Upadyayula et al., 2006; Parvez, 2007; Ci et al., 2012; Nardino et al., 2016a; Öner, 2018).

However, to the best of our knowledge, there are no studies of linear relationships with a higher number of tassel traits in the literature, i.e., with the better technical detail of linear relations between tassel components and grain yield. We assume that such linear relationships exist and can be used for the indirect selection of higher grain yield genotypes. Therefore, this study aimed to verify the linear relationships between grain yield and maize tassel traits and identify traits for selection through direct and indirect effects over three crop years.

\section{Results and discussion}

\section{Analysis of variance and experimental precision}

Individual analyses of variance exhibited significant genotype effects for all traits in the three crop years (Table 1). Meantime, the joint analysis of variance showed a significant genotype effect at a $5 \%$ probability of error for all traits but BSL. Furthermore, only the traits NSB and TBN did not show significant genotype $\times$ crop year interaction (Table 2). These results evidenced differentiated behavior of genotypes in the evaluated years, emphasizing the need to evaluate them in more than one crop year.

Regarding the selective accuracy (SA) among the 36 cases (12 traits $\times$ three crop years), 35 presented very high experimental accuracy $(S A \geq 0.90)$, and only one case, in experiment 1 , displayed high accuracy $(0.70 \leq S A<0.90)$, according to the classification established by Resende and Duarte (2007) (Table 1). When the three experiments were jointly examined, all traits exhibited very high experimental accuracy ( $\mathrm{SA} \geq 0.90$ ) (Table 2). Therefore, given the large dataset (12 traits, 20 genotypes, and three crop years) and the high experimental precision and variability among genotypes, we can infer that the dataset is suitable for studying the linear relationships between tassel traits and grain yield through correlation and path analysis.

The mean values obtained in our study were in agreement with those observed by Upadyayula et al. (2006), who reported similar values for the traits TL, NPB, BSL, CSL, and TDM. Moreover, Andrade and Miranda Filho (2008) observed consonant values for TL, TDM, and TBN. They also agreed with the results described by Nardino et al. (2016b) for TL, NPB, and GY, as well as those observed by Yi et al. (2018) and Öner (2018) for TL and TBN (Table 2). Hence, the mean values of TL, NPB, TBN, BSL, CSL, TDM, and GY display an adequate crop development over the three experiments.

\section{Phenotypic and genotypic correlation coefficients}

Phenotypic and genotypic correlation matrices were similar in the three crop years in the joint analysis (Table 3). Linear associations of PL with the traits BSL, CSL, NPB, NSB, TBN, BSDM, CSDM, TDM, and $\mathrm{GY}$ showed a low magnitude correlation, i.e., between -0.398 and 0.375 , while PL correlations with TL and PDM were significant and of high magnitude, with values ranging from 0.602 to 0.901 . The phenotypic and genotypic correlations of BSL were positive with the traits NPB, NSB, TBN, PDM, BSDM, TDM, and GY. Regarding CSL, the correlations were negative and significant with the traits BSL, NPB, NSB, TBN, and BSDM. The trait TBN correlated positively and significantly with BSL, NPB, NSB, PDM, BSDM, TDM, and GY. For TDM, the phenotypic and genotypic correlations were positive and significant with BSL, NPB, NSB, TBN, PDM, BSDM, and GY. When studying tassel and ear traits, Upadyayula et al. (2006) observed negative and significant correlations between CSL and the traits BSL and TBN. These authors also found a positive and significant association among the traits TBN, BSL and TDM, corroborating the results obtained in our research. Andrade and Miranda Filho (2008) observed low magnitude correlations between TL and the traits TDM and TBN, whereas those between TDM and TBN were positive and of greater magnitude ( $\mathrm{rg}=0.626$ and $r p=0.603$ ), also supporting our results. Lastly, Öner (2018) observed low magnitude correlations of TL with TBN and TDM, which are similar to those in our investigation.

The GY exhibited positive and significant phenotypic and genotypic correlations with the traits NSB, BSDM, and TDM in the first experiment. In the second, it showed a positive and significant correlation with BSL, NPB, NSB, TBN, BSDM, and TDM. And, in the third experiment, the correlation was positive and significant with BSL, TL, NPB, NSB, TBN, BSDM, and TDM. Between CSL and GY, the phenotypic and genotypic correlations were negative and of low magnitude (Table 3). Ci et al. (2012) observed that the association between GY and TL was positive and of low magnitude, corroborating our results as well. However, it is impossible to infer which of the traits has a direct effect on GY only using correlation coefficients. Thus, the unfolding of the coefficients through path analysis is important, as it unfolds direct and indirect effects and reveals the real cause and effect associations among the traits (Wright, 1921).

\section{Diagnosis of multicollinearity}

The diagnosis of the phenotypic and genotypic correlation coefficient matrices among explanatory traits (PL, BSL, CSL, TL, NPB, NSB, TBN, PDM, BSDM, CSDM, and TDM), evaluated in each experiment and jointly, showed a high degree of multicollinearity. Thus, after eliminating the traits PL, CSL, TL, TBN, PDM, CSDM, and BSDM in individual and joint analyses, the correlation matrices exhibited a condition number between 18.19 (phenotypic correlation matrix in experiment 3) and 26.80 (genotype correlation matrix in experiment 1), i.e., weak multicollinearity according to the criteria of Montgomery, Peck, Vinning (2012). Variance inflation factors (VIF) were lower than 10 when the traits PL, CSL, TL, TBN, PDM, CSDM, and BSDM were eliminated, indicating weak multicollinearity (Table 4). Thus, we can infer that the path analyses of the main trait (GY) as a function of the explanatory traits (BSL, NPB, NSB, and TDM) were performed under appropriate conditions (Figure 2).

\section{Path analyzes}

The direct effects of NSB on GY obtained from the phenotypic and genotypic correlation matrices were positive and of high magnitude $(0.501 \leq$ direct effect $\leq 0.855)$, which demonstrates a cause and effect association between these traits. Meantime for TDM on GY, the direct effects were positive but with a lower magnitude $(0.099 \leq$ direct effects $\leq 0.693)$. However, the associations can be explained by the greater indirect effects via NSB. Direct effects were of low magnitude $(-0.482 \leq$ direct effect $\leq$ 0.001 ) for NPB on GY, which can be explained by the high positive indirect effect of NSB on GY $(0.413 \leq$ indirect effects $\leq 0.698)$, featuring the absence of cause-and-effect association between NPB and GY. For BSL on GY, the direct effects were of low magnitude $(-0.402 \leq$ direct effect $\leq 0.412)$. However, the association can be explained by the greater indirect effects via NSB $(0.366 \leq$ indirect effects $\leq 0.698$ ) (Table 4).

Based on the phenotypic and genotypic correlation matrices and path analyses, we can infer that the number of secondary tassel branches had a positive linear association with grain yield. Therefore, it can be used in indirect plant selection for grain yield. In practice, a direct non-destructive selection in the field is feasible after anthesis, by counting the secondary branches without removing tassels from maize plants. 
Table 1. Summary of analysis of variance with degrees of freedom (DF) and mean square for the sources of variation block, genotype and residue, mean, coefficient of variation (CV\%), F-test value for genotype ( $\mathrm{Fc})$, and selective accuracy (AS) for 12 traits in 20 maize genotypes in the 2015-2016 (experiment 1), 2016-2017 (experiment 2) and 2017-2018 (experiment 3) crop years.

\begin{tabular}{|c|c|c|c|c|c|c|c|c|c|c|c|c|c|}
\hline \multirow[t]{2}{*}{ SV } & \multirow[t]{2}{*}{ DF } & \multicolumn{12}{|c|}{ 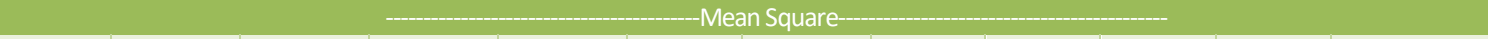 } \\
\hline & & $\mathrm{PL}$ & BSL & CSL & $\mathrm{TL}$ & NPB & NSB & TBN & PDM & BSDM & CSDM & TDM & GY \\
\hline Block & 2 & 0.265 & 0.870 & 0.337 & 3.450 & 3.637 & 0.172 & 5.335 & 0.000 & 0.020 & 0.002 & 0.031 & 0.771 \\
\hline Genotype & 19 & 10.393* & $19.027^{*}$ & $40.976^{*}$ & $24.426^{*}$ & 52.709* & $5.009 *$ & $85.015^{*}$ & $0.022^{*}$ & 1.986* & $0.060 *$ & $2.378^{*}$ & $5.754^{*}$ \\
\hline Residue & 38 & 0.891 & 0.833 & 0.626 & 1.484 & 1.411 & 0.318 & 2.628 & 0.001 & 0.080 & 0.003 & 0.102 & 2.224 \\
\hline Mean & & 8.780 & 12.110 & 26.600 & 47.500 & 11.410 & 2.590 & 14.000 & 0.260 & 2.170 & 0.670 & 3.110 & 9.970 \\
\hline $\mathrm{CV}(\%)$ & & 10.750 & 7.530 & 2.970 & 2.560 & 10.410 & 21.730 & 11.580 & 12.060 & 13.060 & 7.410 & 10.270 & 14.970 \\
\hline Fc & & 11.665 & 22.851 & 65.461 & 16.465 & 37.344 & 15.766 & 32.349 & 22.442 & 24.701 & 23.836 & 23.397 & 2.587 \\
\hline$S A^{(1)}$ & & 0.956 & 0.978 & 0.992 & 0.969 & 0.987 & 0.968 & 0.984 & 0.977 & 0.980 & 0.979 & 0.978 & 0.783 \\
\hline Precision & & VH & VH & VH & VH & VH & VH & VH & VH & VH & VH & VH & $\mathrm{H}$ \\
\hline \multirow[t]{2}{*}{ SV } & \multirow[t]{2}{*}{ DF } & \multicolumn{12}{|c|}{ 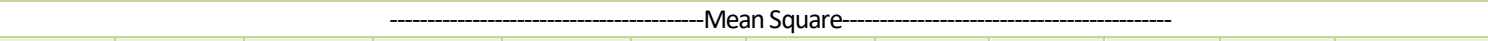 } \\
\hline & & PL & BSL & CSL & TL & NPB & NSB & TBN & PDM & BSDM & CSDM & TDM & GY \\
\hline Block & 2 & 1.063 & 0.463 & 3.465 & 3.193 & 1.451 & 0.293 & 2.949 & 0.003 & 0.011 & 0.007 & 0.053 & 1.849 \\
\hline Genotype & 19 & $12.447^{*}$ & $17.291^{*}$ & $22.092^{*}$ & $35.002 *$ & 33.669* & $3.783^{*}$ & $55.212^{*}$ & $0.022^{*}$ & 0.993* & $0.034^{*}$ & $1.275^{*}$ & $8.455^{*}$ \\
\hline Residue & 38 & 0.414 & 0.611 & 2.032 & 2.402 & 1.317 & 0.199 & 1.857 & 0.000 & 0.022 & 0.002 & 0.034 & 0.959 \\
\hline Mean & & 9.130 & 12.200 & 27.050 & 48.380 & 9.910 & 2.480 & 12.390 & 0.230 & 1.640 & 0.530 & 2.400 & 9.260 \\
\hline $\mathrm{CV}(\%)$ & & 7.040 & 6.410 & 5.270 & 3.200 & 11.580 & 17.980 & 11.000 & 9.180 & 8.960 & 8.080 & 7.700 & 10.570 \\
\hline Fc & & 30.058 & 28.285 & 10.870 & 14.572 & 25.574 & 19.022 & 29.725 & 49.662 & 46.008 & 18.316 & 37.355 & 8.819 \\
\hline$S A^{(1)}$ & & 0.983 & 0.982 & 0.953 & 0.965 & 0.980 & 0.973 & 0.983 & 0.990 & 0.989 & 0.972 & 0.987 & 0.942 \\
\hline Precision & & VH & VH & $\mathrm{VH}$ & VH & VH & VH & VH & VH & VH & VH & VH & VH \\
\hline \multirow[t]{2}{*}{ SV } & \multirow[t]{2}{*}{ DF } & \multicolumn{12}{|c|}{ 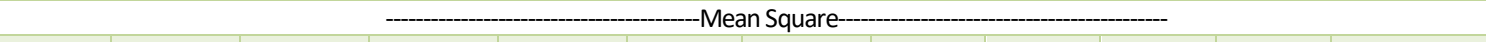 } \\
\hline & & $\mathrm{PL}$ & BSL & CSL & $\mathrm{TL}$ & NPB & NSB & TBN & PDM & BSDM & CSDM & TDM & GY \\
\hline Block & 2 & 0.637 & 0.083 & 5.547 & 8.299 & 0.158 & 0.332 & 0.429 & 0.002 & 0.079 & 0.001 & 0.115 & 2.539 \\
\hline Genotype & 19 & $14.207^{*}$ & $22.506^{*}$ & $33.761^{*}$ & $35.013^{*}$ & $32.862^{*}$ & $3.149 *$ & $52.775^{*}$ & $0.019 *$ & $1.308^{*}$ & $0.056^{*}$ & $1.611^{*}$ & $4.318^{*}$ \\
\hline Residue & 38 & 0.440 & 0.499 & 2.698 & 2.936 & 0.614 & 0.104 & 0.957 & 0.001 & 0.052 & 0.005 & 0.081 & 0.759 \\
\hline Mean & & 7.800 & 12.080 & 24.860 & 44.740 & 9.160 & 2.080 & 11.240 & 0.210 & 1.800 & 0.590 & 2.600 & 8.320 \\
\hline $\mathrm{CV}(\%)$ & & 8.510 & 5.850 & 6.610 & 3.830 & 8.550 & 15.480 & 8.700 & 11.180 & 12.720 & 11.500 & 10.970 & 10.470 \\
\hline Fc & & 32.297 & 45.062 & 12.513 & 11.925 & 53.530 & 30.384 & 55.171 & 32.960 & 25.074 & 12.273 & 19.884 & 5.688 \\
\hline$S A^{(1)}$ & & 0.984 & 0.989 & 0.959 & 0.957 & 0.991 & 0.983 & 0.991 & 0.985 & 0.980 & 0.958 & 0.975 & 0.908 \\
\hline Precision & & VH & VH & VH & VH & VH & VH & VH & VH & VH & VH & VH & VH \\
\hline
\end{tabular}

Traits: PL: peduncle length (considering the distance between the collar of the flag leaf and the first branch), in cm; BSL: branching space length, in cm; CSL: central spike length, in cm; TL: tassel length, in cm; NPB: number of primary branches; NSB: number of secondary branches; TBN: tassel branch number; PDM: peduncle dry matter (considering the region between the flag leaf collar and the first branch); BSDM: branching space dry matter, in g; CSDM: central spike dry matter, in g; TDM: tassel dry matter, in g; and GY: grain yield in Mg ha ${ }^{-1}$ at $13 \%$ moisture. *Significant effect by F-test at $5 \%$ probability of error.

(1) Classification limits for selective accuracy (SA) established by Resende and Duarte (2007): VH: very high $(\mathrm{SA} \geq 0.90), \mathrm{H}$ : high $(0.70 \leq \mathrm{SA}<0.90)$ and $\mathrm{M}$ : moderate $(0.50 \leq \mathrm{SA}<0.70)$.

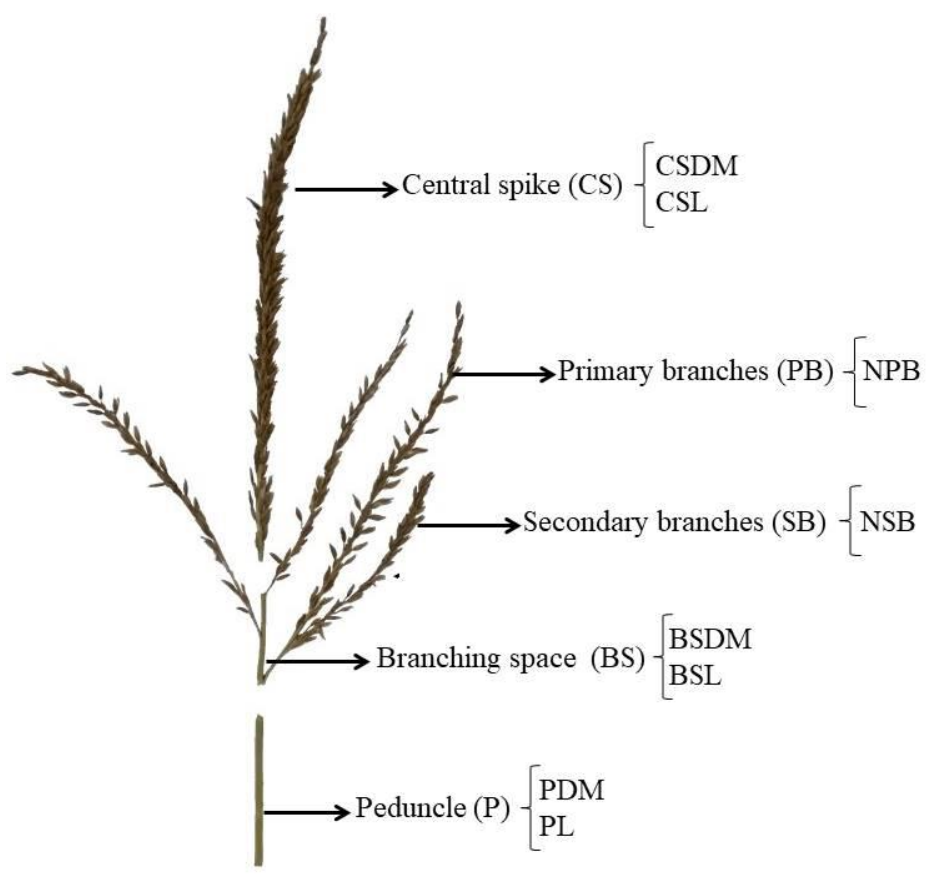

Figure 1. Representation of the traits evaluated in maize tassels, PL: peduncle length (considering the distance between the collar of the flag leaf and the first branch), in cm; BSL: branching space length, in cm; CSL: central spike length, in cm; TL: tassel length, in cm; NPB: number of 
primary branches; NSB: number of secondary branches; PDM: peduncle dry matter (considering the region between the flag leaf collar and the first branch), in g; BSDM: branching space dry matter, in g; CSDM: central spike dry matter, in g. Adapted from Wartha et al. (2016).

Table 2. Summary of joint analysis of variance with degrees of freedom (DF) and mean square for the sources of variation block nested in the crop year, genotype, crop year, genotype $\times$ crop year interaction and residue, mean, coefficient of variation (CV\%), F-test value for genotype (Fc), and selective accuracy (AS) for 12 traits in 20 maize genotypes in the 2015-2016 (experiment 1), 2016-2017 (experiment 2) and 2017-2018 (experiment 3) crop years.

\begin{tabular}{|c|c|c|c|c|c|c|c|}
\hline \multirow[t]{2}{*}{ SV } & \multirow[t]{2}{*}{ DF } & \multicolumn{6}{|c|}{--Mean Square---_-_- } \\
\hline & & PL & BSL & CSL & TL & NPB & NSB \\
\hline Block/Agricultural year & 6 & 0.655 & 0.472 & 3.116 & 4.981 & 1.748 & 0.266 \\
\hline Genotype & 19 & $32.970 *$ & $56.053^{*}$ & $86.068^{*}$ & $80.715^{*}$ & $115.452 *$ & $11.517^{*}$ \\
\hline Agricultural year & 2 & $28.849 *$ & $0.220 \mathrm{~ns}$ & $80.303^{*}$ & $216.841^{*}$ & $78.501^{*}$ & $4.365^{*}$ \\
\hline Genotype $\times$ Agricultural year & 38 & $2.039 *$ & $1.386^{*}$ & $5.380 *$ & $6.862^{*}$ & $1.894^{*}$ & $0.212 \mathrm{~ns}$ \\
\hline Residue & 114 & 0.582 & 0.648 & 1.785 & 2.274 & 1.114 & 0.207 \\
\hline Mean & & 8.570 & 12.131 & 26.172 & 46.874 & 10.161 & 2.385 \\
\hline $\mathrm{CV}(\%)$ & & 8.899 & 6.635 & 5.105 & 3.217 & 10.388 & 19.068 \\
\hline Fc & & 16.173 & 40.450 & 15.997 & 11.762 & 60.951 & 54.357 \\
\hline $\mathrm{SA}^{(1)}$ & & 0.969 & 0.988 & 0.968 & 0.957 & 0.992 & 0.991 \\
\hline Precision & & MA & MA & MA & MA & MA & MA \\
\hline \multirow[t]{2}{*}{ SV } & \multirow[t]{2}{*}{ DF } & \multicolumn{6}{|c|}{ 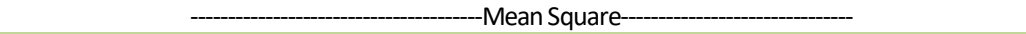 } \\
\hline & & TBN & PDM & BSDM & CSDM & TDM & GY \\
\hline Block/Agricultural year & 6 & 2.904 & 0.002 & 0.036 & 0.003 & 0.066 & 1.720 \\
\hline Genotype & 19 & 187.588* & $0.057^{*}$ & $3.979 *$ & $0.140 *$ & $4.854^{*}$ & $13.936 *$ \\
\hline Agricultural year & 2 & $115.286^{*}$ & $0.032 *$ & $4.475^{*}$ & $0.317^{*}$ & $7.936^{*}$ & $40.765^{*}$ \\
\hline Genotype $\times$ Agricultural year & 38 & 2.707ns & $0.003^{*}$ & $0.154^{*}$ & $0.005^{*}$ & $0.205^{*}$ & $2.296^{*}$ \\
\hline Residue & 114 & 1.814 & 0.001 & 0.051 & 0.003 & 0.072 & 1.314 \\
\hline Mean & & 12.545 & 0.234 & 1.869 & 0.597 & 2.701 & 9.184 \\
\hline CV(\%) & & 10.736 & 10.991 & 12.126 & 9.112 & 9.953 & 12.482 \\
\hline Fc & & 69.306 & 20.199 & 25.822 & 31.141 & 23.697 & 6.070 \\
\hline $\mathrm{SA}^{(1)}$ & & 0.993 & 0.975 & 0.980 & 0.984 & 0.979 & 0.914 \\
\hline Precision & & MA & MA & MA & MA & MA & MA \\
\hline
\end{tabular}

Traits: PL: peduncle length (considering the distance between the collar of the flag leaf and the first branch), in cm; BSL: branching space length, in cm; CSL: central spike length, in cm; TL: tassel length, in cm; NPB: number of primary branches; NSB: number of secondary branches; TBN: tassel branch number; PDM: peduncle dry matter (considering the region between the flag leaf collar and the first branch); BSDM: branching space dry matter, in g; CSDM: central spike dry matter, in g; TDM: tassel dry matter, in g; and GY: grain yield in Mg ha ${ }^{-1}$ at $13 \%$ moisture. *Significant effect by F-test at $5 \%$ probability of error. ns Non-significant.

(1) Classification limits for selective accuracy (SA) established by Resende and Duarte (2007): VH: very high ( $\mathrm{SA} \geq 0.90)$, $\mathrm{H}$ : high $(0.70 \leq \mathrm{SA}<0.90)$ and $\mathrm{M}$ : moderate $(0.50 \leq \mathrm{SA}<0.70)$.

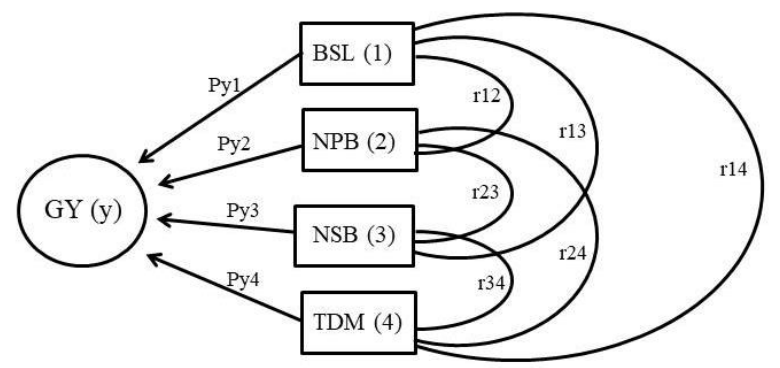

Figure 2. Causal diagram illustrating the direct and indirect effects of explanatory variables: PL: BSL: branching space length, in cm; NPB: number of primary branches; NSB: number of secondary branches; PDM: peduncle dry matter (considering the region between the flag leaf collar and the first branch) on the dependent variable GY: grain yield in $\mathrm{Mg} \mathrm{ha}^{-1}$ at $13 \%$ moisture. Pyi: Direct effect of each of the four explanatory variables on the dependent variable. Rij: correlation coefficient between explanatory variables.

Table 3. Estimates of phenotypic (above diagonal) and genotypic (below diagonal) correlation coefficients among 12 measured traits of 20 maize genotypes evaluated over three crop years.

\begin{tabular}{|c|c|c|c|c|c|c|c|c|c|c|c|c|}
\hline Trait & $\mathrm{PL}$ & BSL & CSL & $\mathrm{TL}$ & NPB & NSB & TBN & PDM & BSDM & CSDM & TDM & GY \\
\hline \multicolumn{13}{|c|}{ Experiment 1 (2015-2016) } \\
\hline $\mathrm{PL}$ & - & 0.359 & -0.284 & $0.602^{*}$ & 0.300 & 0.302 & 0.310 & $0.901^{*}$ & 0.340 & -0.068 & 0.387 & 0.166 \\
\hline BSL & 0.397 & - & $-0.794^{*}$ & 0.088 & $0.794 *$ & $0.839 *$ & $0.828^{*}$ & $0.579 *$ & $0.764^{*}$ & -0.396 & $0.691^{*}$ & 0.354 \\
\hline CSL & -0.303 & $-0.806^{*}$ & - & 0.409 & $-0.812^{*}$ & $-0.789 *$ & $-0.830 *$ & $-0.501^{*}$ & $-0.660 *$ & $0.526^{*}$ & $-0.568^{*}$ & -0.121 \\
\hline TL & $0.595^{*}$ & 0.077 & 0.414 & - & -0.155 & -0.085 & -0.142 & $0.450^{*}$ & 0.041 & 0.288 & 0.126 & 0.264 \\
\hline NPB & 0.327 & $0.796^{*}$ & $-0.818^{*}$ & -0.166 & - & $0.840^{*}$ & 0.991* & $0.515^{*}$ & $0.839 *$ & -0.243 & $0.778^{*}$ & 0.372 \\
\hline NSB & 0.338 & $0.850^{*}$ & $-0.815^{*}$ & -0.106 & $0.851^{*}$ & - & $0.904^{*}$ & $0.451^{*}$ & $0.852^{*}$ & -0.315 & $0.773^{*}$ & $0.540^{*}$ \\
\hline TBN & 0.339 & $0.831^{*}$ & $-0.840^{*}$ & -0.156 & $0.992^{*}$ & $0.910^{*}$ & - & $0.515^{*}$ & $0.868^{*}$ & -0.268 & $0.800^{*}$ & 0.424 \\
\hline PDM & $0.908^{*}$ & $0.605^{*}$ & $-0.519 *$ & 0.435 & $0.535^{*}$ & $0.476^{*}$ & $0.536^{*}$ & - & $0.566^{*}$ & -0.033 & $0.608^{*}$ & 0.230 \\
\hline BSDM & 0.369 & $0.766^{*}$ & $-0.671^{*}$ & 0.029 & $0.843^{*}$ & $0.868^{*}$ & $0.872^{*}$ & $0.583^{*}$ & - & 0.111 & $0.986^{*}$ & $0.531^{*}$ \\
\hline CSDM & -0.079 & -0.412 & $0.535^{*}$ & 0.292 & -0.252 & -0.331 & -0.278 & -0.046 & 0.095 & - & 0.257 & 0.247 \\
\hline TDM & 0.412 & $0.694^{*}$ & $-0.579 *$ & 0.115 & $0.782^{*}$ & $0.787^{*}$ & $0.805^{*}$ & $0.622^{*}$ & $0.986^{*}$ & 0.241 & - & $0.546^{*}$ \\
\hline GY & 0.265 & 0.393 & -0.162 & 0.305 & 0.438 & $0.614^{*}$ & $0.492^{*}$ & 0.324 & $0.656^{*}$ & 0.324 & $0.683^{*}$ & - \\
\hline
\end{tabular}




\begin{tabular}{|c|c|c|c|c|c|c|c|c|c|c|c|c|}
\hline \multicolumn{13}{|c|}{ Experiment 2 (2016-2017) } \\
\hline PL & - & 0.300 & 0.019 & $0.822 *$ & 0.202 & 0.038 & 0.168 & $0.837^{*}$ & 0.229 & 0.000 & 0.313 & -0.053 \\
\hline BSL & 0.319 & - & $-0.672^{*}$ & 0.348 & $0.723^{*}$ & $0.686^{*}$ & $0.744^{*}$ & $0.526^{*}$ & $0.758^{*}$ & -0.382 & $0.676^{*}$ & $0.474^{*}$ \\
\hline CSL & 0.021 & $-0.707^{*}$ & - & 0.334 & $-0.696^{*}$ & $-0.646^{*}$ & $-0.713^{*}$ & -0.134 & $-0.539 *$ & $0.596 *$ & -0.396 & -0.333 \\
\hline TL & 0.853* & 0.355 & 0.291 & - & 0.075 & -0.009 & 0.057 & $0.762 *$ & 0.242 & 0.204 & 0.347 & 0.038 \\
\hline NPB & 0.222 & $0.725^{*}$ & $-0.722 *$ & 0.087 & - & $0.787^{*}$ & $0.987^{*}$ & $0.455^{*}$ & $0.845^{*}$ & -0.083 & $0.792 *$ & $0.614^{*}$ \\
\hline NSB & 0.049 & $0.706^{*}$ & $-0.700 *$ & -0.014 & $0.809 *$ & - & $0.876^{*}$ & 0.156 & $0.788^{*}$ & -0.221 & $0.680^{*}$ & $0.757^{*}$ \\
\hline TBN & 0.185 & $0.748^{*}$ & $-0.743 *$ & 0.064 & $0.988^{*}$ & $0.889 *$ & - & 0.396 & $0.866 *$ & -0.123 & $0.797^{*}$ & $0.677^{*}$ \\
\hline PDM & $0.845^{*}$ & $0.537^{*}$ & -0.151 & $0.779 *$ & $0.470^{*}$ & 0.165 & 0.409 & - & $0.561^{*}$ & 0.144 & $0.650 *$ & 0.124 \\
\hline BSDM & 0.243 & $0.767 *$ & $-0.585 *$ & 0.237 & $0.861^{*}$ & $0.803^{*}$ & $0.878^{*}$ & $0.565 *$ & - & 0.141 & $0.980 *$ & $0.641^{*}$ \\
\hline CSDM & 0.008 & -0.401 & $0.603^{*}$ & 0.191 & -0.088 & -0.232 & -0.128 & 0.138 & 0.126 & - & 0.306 & -0.014 \\
\hline TDM & 0.328 & $0.685^{*}$ & -0.441 & 0.344 & $0.810^{*}$ & $0.695^{*}$ & $0.811^{*}$ & $0.654^{*}$ & $0.980^{*}$ & 0.290 & - & $0.580^{*}$ \\
\hline GY & -0.051 & $0.497^{*}$ & -0.350 & 0.050 & $0.647^{*}$ & $0.804^{*}$ & $0.713^{*}$ & 0.130 & $0.681^{*}$ & -0.006 & 0.619* & - \\
\hline \multicolumn{13}{|c|}{ Experiment 3 (2017-2018) } \\
\hline PL & - & 0.375 & -0.333 & $0.611 *$ & 0.240 & 0.107 & 0.215 & $0.868^{*}$ & -0.009 & -0.398 & 0.012 & 0.219 \\
\hline BSL & 0.387 & - & $-0.622 *$ & 0.430 & 0.749* & 0.731* & $0.769 *$ & $0.595^{*}$ & $0.682^{*}$ & -0.353 & $0.613^{*}$ & $0.641^{*}$ \\
\hline CSL & -0.345 & $-0.649 *$ & - & 0.271 & $-0.615^{*}$ & $-0.545^{*}$ & $-0.619^{*}$ & -0.304 & -0.223 & $0.794^{*}$ & -0.086 & -0.200 \\
\hline $\mathrm{TL}$ & $0.636^{*}$ & 0.443 & 0.220 & - & 0.149 & 0.119 & 0.147 & $0.732^{*}$ & 0.323 & 0.244 & 0.415 & $0.457^{*}$ \\
\hline NPB & 0.248 & $0.752^{*}$ & $-0.629 *$ & 0.166 & - & $0.824^{*}$ & $0.990^{*}$ & 0.422 & $0.786^{*}$ & -0.195 & $0.718^{*}$ & $0.538^{*}$ \\
\hline NSB & 0.116 & $0.741^{*}$ & $-0.547^{*}$ & 0.152 & $0.834^{*}$ & - & $0.895^{*}$ & 0.248 & $0.816^{*}$ & -0.211 & $0.723^{*}$ & $0.665^{*}$ \\
\hline TBN & 0.224 & $0.773^{*}$ & $-0.629 *$ & 0.168 & $0.991 *$ & $0.900^{*}$ & - & 0.394 & $0.820 *$ & -0.205 & $0.743^{*}$ & $0.587^{*}$ \\
\hline PDM & $0.877^{*}$ & $0.608^{*}$ & -0.336 & $0.747^{*}$ & 0.437 & 0.264 & 0.409 & - & 0.333 & -0.176 & 0.375 & 0.379 \\
\hline BSDM & 0.001 & $0.692^{*}$ & -0.270 & 0.308 & $0.807^{*}$ & $0.850^{*}$ & 0.843* & 0.331 & - & 0.251 & $0.984^{*}$ & $0.573^{*}$ \\
\hline CSDM & -0.402 & -0.364 & $0.793^{*}$ & 0.216 & -0.189 & -0.203 & -0.198 & -0.195 & 0.230 & - & 0.393 & -0.094 \\
\hline TDM & 0.023 & $0.627^{*}$ & -0.136 & 0.400 & $0.744^{*}$ & $0.762 *$ & $0.772 *$ & 0.373 & $0.984 *$ & 0.370 & - & $0.539 *$ \\
\hline GY & 0.257 & $0.690^{*}$ & -0.244 & $0.500^{*}$ & 0.589* & $0.717^{*}$ & $0.639 *$ & 0.424 & $0.620^{*}$ & -0.119 & $0.587^{*}$ & - \\
\hline \multicolumn{13}{|c|}{ General } \\
\hline PL & - & 0.352 & -0.197 & $0.729 *$ & 0.251 & 0.143 & 0.233 & 0.871* & 0.181 & -0.178 & 0.228 & 0.104 \\
\hline BSL & 0.355 & - & $-0.746 *$ & 0.288 & $0.762^{*}$ & $0.768^{*}$ & $0.788^{*}$ & $0.575^{*}$ & 0.735* & -0.396 & $0.660^{*}$ & $0.559 *$ \\
\hline CSL & -0.187 & $-0.775 *$ & - & 0.285 & $-0.752^{*}$ & $-0.712^{*}$ & $-0.766^{*}$ & -0.344 & $-0.541^{*}$ & $0.643^{*}$ & -0.418 & -0.294 \\
\hline TL & $0.757^{*}$ & 0.280 & 0.258 & - & 0.020 & -0.004 & 0.014 & $0.681^{*}$ & 0.169 & 0.220 & 0.264 & 0.228 \\
\hline NPB & 0.255 & $0.768^{*}$ & $-0.771^{*}$ & 0.019 & - & $0.831^{*}$ & 0.991* & $0.478^{*}$ & 0.839* & -0.186 & $0.780^{*}$ & $0.593 *$ \\
\hline NSB & 0.139 & $0.777^{*}$ & $-0.735^{*}$ & -0.010 & $0.838 *$ & - & $0.900 *$ & 0.290 & $0.842 *$ & -0.259 & $0.750 *$ & $0.749 *$ \\
\hline TBN & 0.234 & $0.794 *$ & $-0.786 *$ & 0.013 & 0.991* & $0.904 *$ & - & $0.446 *$ & $0.867^{*}$ & -0.210 & $0.797^{*}$ & $0.651^{*}$ \\
\hline PDM & $0.875^{*}$ & $0.581^{*}$ & -0.352 & $0.697^{*}$ & $0.484 *$ & 0.288 & $0.450 *$ & - & 0.483* & -0.046 & 0.539* & 0.279 \\
\hline BSDM & 0.179 & 0.739* & $-0.572^{*}$ & 0.154 & $0.847^{*}$ & $0.854^{*}$ & $0.875^{*}$ & $0.481^{*}$ & - & 0.151 & 0.983* & $0.685^{*}$ \\
\hline CSDM & -0.181 & -0.408 & $0.646^{*}$ & 0.207 & -0.187 & -0.261 & -0.211 & -0.054 & 0.143 & - & 0.301 & 0.041 \\
\hline TDM & 0.226 & $0.664 *$ & $-0.446 *$ & 0.251 & $0.789 *$ & $0.761^{*}$ & $0.806^{*}$ & 0.535* & 0.983* & 0.294 & - & $0.657^{*}$ \\
\hline GY & 0.111 & $0.617^{*}$ & -0.355 & 0.231 & $0.662 *$ & $0.820^{*}$ & $0.722^{*}$ & 0.316 & 0.771* & 0.033 & 0.739* & - \\
\hline
\end{tabular}

Table 4. Path analysis direct and indirect effects of the phenotypic and genotypic correlation matrices of explanatory traits on grain yield (GY) of 20 maize genotypes evaluated in three crop years.

\begin{tabular}{|c|c|c|c|c|c|c|c|c|}
\hline \multirow[t]{2}{*}{ Effects } & \multicolumn{4}{|c|}{ Phenotypic correlation matrix } & \multicolumn{4}{|c|}{ Genotypic correlation matrix } \\
\hline & BSL & NPB & NSB & TDM & BSL & NPB & NSB & TDM \\
\hline & \multicolumn{8}{|c|}{ Experiment 1 (2015/2016) } \\
\hline Direct on GY & -0.286 & -0.402 & 0.748 & 0.479 & -0.402 & -0.482 & 0.821 & 0.693 \\
\hline Indirect via BSL & & -0.227 & -0.240 & -0.198 & & -0.320 & -0.342 & -0.279 \\
\hline Indirect via NPB & -0.319 & & -0.338 & -0.313 & -0.384 & & -0.410 & -0.377 \\
\hline Indirect via NSB & 0.628 & 0.628 & & 0.578 & 0.698 & 0.698 & & 0.646 \\
\hline Indirect via TDM & 0.331 & 0.373 & 0.370 & & 0.481 & 0.542 & 0.545 & \\
\hline Total & $0.354 n s$ & $0.372 \mathrm{~ns}$ & $0.540^{*}$ & $0.546^{*}$ & $0.393 n s$ & $0.438 \mathrm{~ns}$ & $0.614^{*}$ & $0.683^{*}$ \\
\hline Coefficient of determination & \multicolumn{4}{|l|}{0.415} & \multicolumn{4}{|l|}{0.608} \\
\hline Condition number & \multicolumn{4}{|l|}{23.61} & \multicolumn{4}{|l|}{26.80} \\
\hline Maximum variance inflation factor & 3.713 & 4.275 & 5.118 & 2.885 & 3.876 & 4.395 & 5.761 & 2.991 \\
\hline \multirow[t]{3}{*}{ Effects } & \multicolumn{4}{|c|}{ Phenotypic correlation matrix } & \multicolumn{4}{|c|}{ Genotypic correlation matrix } \\
\hline & BSL & NPB & NSB & TDM & BSL & NPB & NSB & TDM \\
\hline & \multicolumn{8}{|c|}{ Experiment 2 (2016/2017) } \\
\hline Direct on GY & -0.158 & 0.001 & 0.740 & 0.183 & -0.205 & -0.086 & 0.855 & 0.234 \\
\hline Indirect via BSL & & -0.114 & -0.109 & -0.107 & & -0.148 & -0.144 & -0.140 \\
\hline Indirect via NPB & 0.001 & & 0.001 & 0.001 & -0.062 & & -0.069 & -0.069 \\
\hline Indirect via NSB & 0.507 & 0.582 & & 0.503 & 0.604 & 0.692 & & 0.594 \\
\hline Indirect via TDM & 0.124 & 0.145 & 0.125 & & 0.160 & 0.190 & 0.163 & \\
\hline Total & $0.474^{*}$ & $0.614^{*}$ & $0.757^{*}$ & $0.580^{*}$ & $0.497^{*}$ & $0.647^{*}$ & $0.804^{*}$ & $0.619 *$ \\
\hline Coefficient of determination & \multicolumn{4}{|l|}{0.592} & \multicolumn{4}{|l|}{0.675} \\
\hline Condition number & \multicolumn{4}{|l|}{18.42} & \multicolumn{4}{|l|}{21.28} \\
\hline Maximum variance inflation factor & 2.374 & 4.123 & 2.861 & 2.883 & 2.426 & 4.582 & 3.173 & 3.097 \\
\hline \multirow[t]{3}{*}{ Effects } & \multicolumn{4}{|c|}{ Phenotypic correlation matrix } & \multicolumn{4}{|c|}{ Genotypic correlation matrix } \\
\hline & BSL & NPB & NSB & TDM & BSL & NPB & NSB & TDM \\
\hline & \multicolumn{8}{|c|}{ Experiment 3 (2017/2018) } \\
\hline
\end{tabular}




\begin{tabular}{|c|c|c|c|c|c|c|c|c|}
\hline Direct on GY & 0.391 & -0.253 & 0.501 & 0.119 & 0.412 & -0.245 & 0.540 & 0.099 \\
\hline Indirect via BSL & & 0.293 & 0.286 & 0.240 & & 0.310 & 0.305 & 0.258 \\
\hline Indirect via NPB & -0.189 & & -0.208 & -0.181 & -0.184 & & -0.204 & -0.182 \\
\hline Indirect via NSB & 0.366 & 0.413 & & 0.362 & 0.400 & 0.450 & & 0.411 \\
\hline Indirect via TDM & 0.073 & 0.085 & 0.086 & & 0.062 & 0.074 & 0.075 & \\
\hline Total & $0.641^{*}$ & $0.538 *$ & $0.665^{*}$ & $0.539 *$ & $0.690 *$ & $0.589 *$ & $0.717^{*}$ & $0.587^{*}$ \\
\hline Coefficient of determination & \multicolumn{4}{|l|}{0.512} & \multicolumn{4}{|l|}{0.585} \\
\hline Condition number & \multicolumn{4}{|l|}{18.19} & \multicolumn{4}{|l|}{19.58} \\
\hline Maximum variance inflation factor & 2.519 & 3.919 & 3.772 & 2.331 & 2.555 & 4.086 & 4.163 & 2.629 \\
\hline \multirow[t]{3}{*}{ Effects } & \multicolumn{4}{|c|}{ Phenotypic correlation matrix } & \multicolumn{4}{|c|}{ Genotypic correlation matrix } \\
\hline & BSL & NPB & NSB & TDM & BSL & NPB & NSB & TDM \\
\hline & \multicolumn{8}{|l|}{ General } \\
\hline Direct on GY & -0.030 & -0.239 & 0.738 & 0.311 & -0.044 & -0.254 & 0.785 & 0.372 \\
\hline Indirect via BSL & & -0.023 & -0.023 & -0.020 & & -0.034 & -0.034 & -0.029 \\
\hline Indirect via NPB & -0.182 & & -0.199 & -0.186 & -0.195 & & -0.213 & -0.200 \\
\hline Indirect via NSB & 0.566 & 0.613 & & 0.553 & 0.609 & 0.657 & & 0.597 \\
\hline Indirect via TDM & 0.205 & 0.242 & 0.233 & & 0.247 & 0.293 & 0.283 & \\
\hline Total & $0.559^{*}$ & $0.593 *$ & $0.749 *$ & $0.657^{*}$ & $0.617^{*}$ & $0.662^{*}$ & $0.820^{*}$ & $0.739 *$ \\
\hline Coefficient of determination & \multicolumn{4}{|l|}{0.598} & \multicolumn{4}{|l|}{0.723} \\
\hline Condition number & \multicolumn{4}{|l|}{19.82} & \multicolumn{4}{|l|}{20.71} \\
\hline Maximum variance inflation factor & 2.780 & 4.331 & 4.013 & 2.799 & 2.855 & 4.495 & 4.216 & 2.909 \\
\hline
\end{tabular}

Table 5. Version, technology, company, type, cycle, use, kernel, color and investment of 20 maize genotypes.

\begin{tabular}{|c|c|c|c|c|c|c|c|c|c|}
\hline Hybrid & Version & Technology ${ }^{(1)}$ & Company & Type $^{(2)}$ & Cycle & $U_{s e}^{(3)}$ & Kernel & Color $^{(4)}$ & Investment \\
\hline $20 A 55$ & PW & PowerCore & Morgan Seeds & $\mathrm{TH}$ & $\mathrm{E}$ & $\mathrm{G} / \mathrm{S}$ & Semi-flint & LO & Medium \\
\hline 30F53 & $\mathrm{YH}$ & Optimum Intrasect & Pioneer & SH & $\mathrm{E}$ & $\mathrm{G} / \mathrm{S}$ & Semi-dent & 0 & High \\
\hline AG8780 & PRO 3 & VTPRO 3 & Agroceres seeds & SH & $\mathrm{E}$ & G & Semi-dent & LO & High \\
\hline BM3066 & PRO2 & VT PRO 2 & Biomatrix & SH & $\mathrm{E}$ & $\mathrm{G} / \mathrm{S}$ & Semi-dent & 0 & High \\
\hline DKB 290 & $\mathrm{PRO} 3$ & VT PRO 3 & Dekalb & SH & $\mathrm{E}$ & G & Semi-dent & LO & High \\
\hline MS 2010 & - & Conventional & Melhoramento Agropastoril & SH & $\mathrm{E}$ & G & Semi-dent & Y/LO & High \\
\hline MS 2013 & - & Conventional & Melhoramento Agropastoril & SH & $\mathrm{E}$ & G & Semi-flint & LO & High \\
\hline MS 3022 & - & Conventional & Melhoramento Agropastoril & $\mathrm{TH}$ & $\mathrm{E}$ & G & Flint & 0 & Medium \\
\hline Status & VIP & Agrisure Viptera & Syngenta Seeds & SH & $\mathrm{E}$ & G & Flint & LO & High \\
\hline SX7331 & VIP & Agrisure Viptera & Syngenta Seeds & SH & $\mathrm{E}$ & G & Flint & 0 & High \\
\hline 30A68 & PW & PowerCore & Morgan Seeds & SH & SE & G & Semi-flint & LO & High \\
\hline AG9025 & $\mathrm{PRO} 3$ & VTPRO 3 & Agroceres seeds & SH & SE & G & Semi-dent & LO & High \\
\hline AM9724 & - & Conventional & Melhoramento Agropastoril & SH & SE & G & Dent & Y/LO & High \\
\hline AS1666 & $\mathrm{PRO} 3$ & VTPRO 3 & Agroeste & SH & SE & G & Semi-dent & Y/LO & High \\
\hline AS1677 & PRO 3 & VT PRO 3 & Agroeste & SH & SE & G & Semi-dent & LO & High \\
\hline Celeron & $\mathrm{TL}$ & Agrisure TL & Syngenta Seeds & SH & SE & G & Flint & LO & High \\
\hline DKB 230 & PRO 3 & VT PRO 3 & Dekalb & SH & SE & G & Semi-dent & $\mathrm{Y}$ & High \\
\hline P1630 & $\mathrm{H}$ & HerculexI & Pioneer & SH & SE & G & Semi-dent & LO & High \\
\hline P2530 & - & Conventional & Pioneer & SH & SE & G & Semi-flint & 0 & High \\
\hline SHS 7915 & PRO & YieldGard VT PRO & Santa Helena Seeds & SH & SE & $\mathrm{G} / \mathrm{S}$ & Semi-flint & LO & High \\
\hline
\end{tabular}

\section{Materials and methods}

\section{Conduction of study and experimental design}

Three experiments were conducted with maize in the 2015/2016 (experiment 1), 2016/2017 (experiment 2), and 2017/2018 (experiment 3) crop years. According to the Köppen climate classification, the climate in the region is defined as Cfa, which stands for humid subtropical, with hot summers and no dry season defined (Alvares et al., 2013). The soil of the area is classified as sandy-loam typic Paleudalf (Santos et al., 2018). The experiments were constituted of 20 genotypes from a network of evaluation trials of maize cultivars in the state of Rio Grande do Sul, which is coordinated by the State Foundation for Agricultural Research (FEPAGRO) (Table 5).

The experimental design used was randomized complete blocks with three replicates. Plots consisted of two 5-m-long rows spaced $0.80 \mathrm{~m}$ apart and $0.20 \mathrm{~m}$ between plants within rows, totaling a useful area of $8 \mathrm{~m}^{2}$ per plot. The experiments were conducted for three years, using the same hybrids, which are grown commercially on a large scale. In this way, consistent data and results were obtained.

\section{Cultural management}

Sowings were carried out on Oct/21/2015, Nov/19/2016, and Oct/31/2017 respectively for experiments 1,2 , and 3 . Basal dressing was performed along with sowing in the three experiments. In the 2015/2016 crop year (experiment 1), an NPK 05-20-20 commercial formulation was used to provide $37.5 \mathrm{~kg}$ ha ${ }^{1} \mathrm{~N}, 150 \mathrm{~kg} \mathrm{ha}^{-1} \mathrm{P}_{2} \mathrm{O}_{5}$, and $150 \mathrm{~kg} \mathrm{ha}^{-1} \mathrm{~K}_{2} \mathrm{O}$, and topdressing fertilization with $121.5 \mathrm{~kg} \mathrm{ha}^{-1} \mathrm{~N}$ in the form of urea into three splits. In the 2016/2017 crop year (experiment 2), an NPK 05-2020 commercial formulation was used to provide $20 \mathrm{~kg} \mathrm{ha}^{-1} \mathrm{~N}, 80$ $\mathrm{kg} \mathrm{ha}^{-1} \mathrm{P}_{2} \mathrm{O}_{5}$, and $80 \mathrm{~kg} \mathrm{ha}^{-1} \mathrm{~K}_{2} \mathrm{O}$, and topdressing with $180 \mathrm{~kg} \mathrm{ha}^{-1}$ $\mathrm{N}$ in the form of urea into two splits. In the 2017/2018 crop year (experiment 3), an NPK 05-20-20 commercial formulation was used to provide $15 \mathrm{~kg} \mathrm{ha}^{-1} \mathrm{~N}, 60 \mathrm{~kg} \mathrm{ha}^{-1} \mathrm{P}_{2} \mathrm{O}_{5}$, and $60 \mathrm{~kg} \mathrm{ha}^{-1} \mathrm{~K}_{2} \mathrm{O}$, and topdressing with $157.5 \mathrm{~kg} \mathrm{ha}^{-1} \mathrm{~N}$ in the form of urea into two splits. Plant density was adjusted by manual thinning to five plants 
per meter in each row, totaling 62,500 plants per hectare. The cultural practices were carried out according to the recommendations for maize cropping, maintaining the experimental area in a competition-free condition of weeds, pests, and diseases (Fancelli and Dourado Neto, 2009).

\section{Data collection}

When plants were at the reproductive stage, we randomly collected 20, 11, and 20 tassels per plot respectively in the 2015/2016, 2016/2017, and 2017/2018 crop years. As reported by Wartha et al. (2016), sample sizes containing $11,20,43$, and 169 tassels are enough to estimate averages of tassel traits at precision levels of $40,30,20$, and $10 \%$, respectively (for a $95 \%$ confidence level). After being collected in the field, tassels were identified, stored in paper packaging, and dried in a forced-air ventilation oven $(60$ ㅇ) until a constant weight was reached. The following traits were measured for each tassel: peduncle length ( $\mathrm{PL}$, considering the distance between the collar of the flag leaf and the first branch), in $\mathrm{cm}$; branching space length (BSL), in cm; central spike length (CSL), in cm; tassel length ( $T L=P L+B S L+C S L)$, in $\mathrm{cm}$; number of primary branches (NPB); number of secondary branches (NSB); tassel branch number (TBN=NPB+NSB); peduncle dry matter (PDM, considering the region between the flag leaf collar and the first branch), in g; branching space dry matter (BSDM), in g; central spike dry matter (CSDM), in g; and tassel dry matter (TDM=PDM+BSDM+CSDM), in $\mathrm{g}$ (Figure 1). All plant in the plot were assessed for grain yield (GY), which was expressed as $\mathrm{Mg} \mathrm{ha}^{-1}$ at $13 \%$ moisture.

\section{Statistical analyses}

The Kolmogorov-Smirnov and Levene tests were used to verify the normality of errors and homogeneity of residual variances for all 12 traits measured, respectively. Subsequently, individual and joint analyses of variance were performed at a $5 \%$ level of significance. For the joint analysis, the effects of genotypes were considered fixed, and crop years deemed as random. Estimates of the means, coefficient of variation (CV), F-calculated for genotype $(\mathrm{Fc})$, and selective accuracy $(\mathrm{SA})$ were recorded for each trait (PL, BSL, CSL, TL, NPB, NSB, TBN, PDM, BSDM, CSDM, TDM, and GY), as described by Resende and Duarte (2007). Linear relationships were investigated using estimated matrices of phenotypic ( $r p$ ) and genotypic $(\mathrm{rg})$ correlation coefficients among the traits with significant differences by individual variance analysis (F-test) and by joint variance analysis. Besides, the significance of phenotypic and genotypic correlation coefficients was tested as per the Student's t-test at a $5 \%$ probability of error. The multicollinearity of the phenotypic and genotypic correlation matrices was diagnosed by a variance inflation factor (VIF) and condition number (CN), as established by Montgomery, Peck and Vinning (2012). The criteria used were VIF $<10$ and $\mathrm{CN}<100$ as indicative of weak multicollinearity. In the presence of a high degree of multicollinearity (detrimental to path analysis), one or more traits highly correlated were excluded. A path analysis was performed for each experiment (crop year), considering grain yield as the main trait and tassel traits as explanatory. Additionally, a general path analysis (all experiments) was also performed. The path analyses enabled identifying explanatory traits with direct and indirect effects on the main trait. Statistical analyses were performed using the Genes software (Cruz, 2016) and Microsoft Office Excel ${ }^{\circledR}$ application.

\section{Conclusions}

The number of secondary tassel branches had a positive linear relationship with grain yield and can be used for the indirect selection of maize plants in breeding programs.

\section{Acknowledgments}

We thank the Brazilian National Council for Scientific and Technological Development (CNPq - Processis no. 146258/2019-3; 304652/2017-2) and the Coordination for the Improvement of Higher Education Personnel (CAPES) for granting scholarships.

\section{References}

Alvares CA, Stape JL, Sentelhas PC, Gonçalves JLM, Sparovek G (2013) Köppen's climate classification map for Brazil. Meteorol Z. 22 (6): 711-728.

Andrade JAC, Miranda Filho JB (2008) Quantitative variation in the tropical maize population, ESALQ-PB1.. Sci Agric. 65 (2): 174182.

Brewbaker JL (2015) Diversity and genetics of tassel branch numbers in maize. Crop Sci. 55: 65-78.

Ci X, Li M, Xu J, Lu Z, Bai P, Ru G, Liang X, Zhang D, Li X, Bai L, Xie C, Hao Z, Zhang S, Dong, S (2012) Trends of grain yield and plant traits in Chinese maize cultivars from the 1950s to the 2000. Euphytica. 185: 395-406.

Cruz CD, Carneiro PCS, Regazzi AJ (2014) Modelos biométricos aplicados ao melhoramento genético. 5 rd edn Viçosa: UFV.

Cruz CD (2016) Genes Software - extended and integrated with the R, Matlab and Selegen. Acta Sci-Agron. 38 (4): 547-552.

Duvick DN, Smith JSC, Cooper M (2004) Long-term selection in a commercial hybrid maize breeding program. In: Plant Breeding Reviews. Part 2: Long-term selection: crops, animals, and bacteria. 1rd edn. John Wiley \& Sons, New Jersey. 109-152.

Duvick D (2005) Genetic progress in yield of United States maize (Zea mays L.). Maydica. 50 (3): 193-202.

Edwards J (2011) Changes in plant morphology in response to recurrent selection in the iowa stiff stalk synthetic maize population. Crop Sci. 51 (6): 2352-2361.

Fischer RA, Edmeades GO (2010) Breeding and cereal yield progress. Crop Sci. 50: 85-98.

Fancelli AL, Dourado Neto D (2009) Milho: manejo e produtividade. 1rd edn. ESALQ/USP, Piracicaba. 181.

Gage JL, White MR, Edwards JW, Kaeppler S, De Leon N (2018) Selection signatures underlying dramatic male inflorescence transformation during modern hybrid maize breeding. Genetics. 210 (3): 1125-1138.

Montgomery DC, Peck EA, Vinning GG (2012) Introduction to linear regression analysis. 5rd edn. Wiley, New York. 672.

Nardino M, De Souza VQ, Baretta D, Konflanz VA, Carvalho IC, Follmann DN, Caron BO (2016a) Association of secondary traits with yield in maize F 1 's. Cienc Rural. 46 (5): 776-782.

Nardino M, De Souza VQ, Baretta D, Konflanz VA, Follmann DN, Carvalho IC, Ferrari M, Caron BO, Schmidt, D (2016b) Partial diallel analysis among maize lines for characteristics related to the tassel and the productivity. Afr J Agric Res. 11 (11): 974-982.

Öner F (2018) Assessment of genetic variation in turkish local maize genotypes using multivariate discriminant analysis. Appl Ecol Env Res. 16 (2): 1369-1380.

Parvez AS (2007) Genetic Analysis of Tassel and Ear Characters in Maize (Zea mays L.) Using Triple Test Cross. Asian J Plant Sci. 6 (5): 881-883.

Porto APF, Vasconcelos RC, Viana AES, Almeida MRS (2011) Variedades de milho a diferentes espaçamentos no Planalto de Vitória da Conquista - BA. Rev. Bras. Cienc. Agrar. 6 (2): 208-214. Resende MDV, Duarte JB (2007) Precisão e controle de qualidade em experimentos de avaliação de cultivares. Pesq. Agropec. Trop. 37 (3): 182-194. 
Santos HG, Jacomine PKT, Anjos LHC, Oliveira VA, Lumbreras JF, Coelho MR, Almeida JA, Cunha, TJF, Oliveira JB (2013) Sistema brasileiro de classificação de solos. 5rd edn. Brasília: Embrapa.

Wartha CA, Cargnelutti Filho A, Lúcio AD, Follmann DN, Kleinpaul JA, Simões FM (2016) Sample sizes to estimate mean values for tassel traits in maize genotypes. Genet Mol Res. 15 (4): 1-13.

Wright S (1921) Correlation and causation. J Agric Res.20, 557585.

Upadyayula N, Da Silva HS, Bohn MO, Rocheford TR (2006) Genetic and QTL analysis of maize tassel and ear inflorescence architecture. Theor Appl Genet. 112: 592-606.
Yi Q, Liu Y, Zhang X, Hou X, Zhang J, Liu H, Hu Y, Yu G, Huang, Y (2018) Comparative mapping of quantitative trait loci for tasselrelated traits of maize in F2:3 and RIL populations. J Genet. 97: 253-266. 